\title{
CORRELATIONS AND FLUCTUATIONS (AN INTRODUCTORY REVIEW)
}

\author{
WOLFRAM KITTEL \\ HEFIN, University of Nijmegen/NIKHEF, Toernooiveld 1, 6515 ED Nijmegen, NL \\ E-mail: wolfram@hef.kun.nl
}

\begin{abstract}
Far too often are multiparticle final states studied and models tested on merely single-particle spectra and their integrals, the average multiplicities: A multiparticle final state is a non-linear, complex system and the essential information on the dynamics is contained in the particle correlations and their integrals measuring the fluctuations!
\end{abstract}

\section{The formalism}

We start by defining symmetrized inclusive $q$-particle distributions

$$
\rho_{q}\left(p_{1}, \ldots, p_{q}\right)=\sigma_{\text {tot }}^{-1} \mathrm{~d} \sigma_{q}\left(p_{1}, \ldots, p_{q}\right) / \prod_{1}^{q} \mathrm{~d} p_{q}
$$

where $\sigma_{q}\left(p_{1}, \ldots, p_{q}\right)$ is the inclusive cross section for $q$ particles to be at $p_{1}, \ldots, p_{q}$, irrespective of the presence and location of any further particles, $p_{i}$ is the (four-) momentum of particle $i$ and $\sigma_{\text {tot }}$ is the total hadronic cross section of the collision under study. For the case of identical particles, integration over an interval $\Omega$ in $p$-space yields

$$
\int_{\Omega} \mathrm{d} p_{1} \ldots \int_{\Omega} \mathrm{d} p_{q} \rho_{q}\left(p_{1}, \ldots, p_{q}\right)=\langle n(n-1) \ldots(n-q+1)\rangle,
$$

where $n$ is the multiplicity of identical particles within $\Omega$ in a given event and the angular brackets imply the average over the event ensemble.

Besides the interparticle correlations, the $\rho_{q}$ in general contain "trivial" contributions from lower-order densities. We, therefore, consider a sequence of cumulant coorelation functions $C_{q}\left(p_{1}, \ldots, p_{q}\right)$ which vanish whenever one of their arguments becomes statistically independent of the others 1

$$
\begin{aligned}
C_{2}\left(p_{1}, p_{2}\right) & =\rho_{2}\left(p_{1}, p_{2}\right)-\rho_{1}\left(p_{1}\right) \rho_{1}\left(p_{2}\right), \\
C_{3}\left(p_{1}, p_{2}, p_{3}\right) & =\rho_{3}\left(p_{1}, p_{2}, p_{3}\right)-\sum_{(3)} \rho_{1}(1) \rho_{2}\left(p_{2}, p_{3}\right)+2 \rho_{1}\left(p_{1}\right) \rho_{1}(2 p) \rho_{1}\left(p_{3}\right),
\end{aligned}
$$

etc. Deviations of these functions from zero shall be addressed as genuine correlations. For recent discussions, it is important to note that not the $\rho_{q}$ but the $C_{q}$ are additive in case of $N$ independent overlapping samples. 
It is often convenient to normalize the functions $\rho_{q}$ and $C_{q}$ by the uncorrelated densities,

$$
\begin{aligned}
& R_{q}\left(p_{1}, \ldots, p_{q}\right)=\rho_{q}\left(p_{q}, \ldots, p_{q}\right) / \rho_{1}\left(p_{1}\right) \ldots \rho_{1}\left(p_{q}\right), \\
& K_{q}\left(p_{1}, \ldots, p_{q}\right)=C_{q}\left(p_{1}, \ldots, p_{q}\right) / \rho_{1}\left(p_{1}\right) \ldots \rho_{1}\left(p_{q}\right) .
\end{aligned}
$$

Important relations between the two sets of functions are:

$$
R_{2}=1+K_{2} ; \quad R_{3}=1+3 K_{2}+K_{3}
$$

Because of the additivity of $C_{q}$, the $K_{q}$ will show a dilution according to $K_{q}^{(N)}=N^{(1-q)} K_{q}^{(1)}$ for $N$ independent overlapping sources.

Correlations have been studied extensively for $q=2$ and a strong positive correlation is found for $p_{1} \approx p_{2}$.2 To be able to do that for $q \geq 3$, one must, however, work via the integrals Eq.(2), in limited phase-space cells.

In practical work, with limited statistics, it is almost always necessary to perform averages over more than a single phase-space cell. Let $\Omega_{m}$ be such a cell (e.g. a single rapidity interval of size $\delta y$ ) and divide the phasespace volume $\Omega$ into $M$ non-overlapping equal cells $\Omega_{m}$ of size $\delta \Omega=\Omega / M$. Normalized cell-averaged factorial moments $\mathbf{B}$ are then defined as

$F_{q}(\delta \Omega) \equiv \frac{1}{M} \sum_{m=1}^{M} \frac{\int_{\delta \Omega} \rho_{q}\left(p_{1}, \ldots, p_{q}\right) \prod_{i=1}^{q} \mathrm{~d} p_{i}}{\left(\int_{\delta y} \rho(p) \mathrm{d} p\right)^{q}}=\frac{1}{M} \sum_{m=1}^{M} \frac{\left\langle n_{m}\left(n_{m}-1\right) \ldots\left(n_{m}-q+1\right)\right\rangle}{\left\langle n_{m}\right\rangle^{q}}$.

Likewise, cell-averaged normalized factorial cumulant moments $K_{q}(\delta y)$ may be defined 4 by replacing the $\rho_{q}$ by $C_{q}$ in (6). In analogy to Eq. (5), they are related to the factorial moments by

$$
F_{2}=1+K_{2} \quad ; \quad F_{3}=1+3 K_{2}+K_{3} \quad .
$$

Essential porperties are:

1. The Poisson-noise suppression of the $F_{q}$, contrary to e.g. $\left\langle n^{q}\right\rangle /\langle n\rangle^{q}$.

2. Independent emission leads to $F_{q} \equiv 1$ and $K_{q} \equiv 0$ for all $q$.

3 . The factorial moments resolve the high- $n_{m}$ tail of the multiplicity distribution and are, therefore, sensitive to high-density fluctuations.

A fruitful further development 5 is the use of integrals of $\rho_{q}$ or $C_{q}$ over a strip domain, rather than a sum of the box domains. One not only avoids unwanted side-effects such as splitting of density spikes, but also drastically increases the statistical significance at a given resolution. It, furthermore, allows to work in terms of inter-particle distance measures, as the (invariant) four-momentum difference $Q=\sqrt{-\left(p_{1}-p_{2}\right)^{2}}$.

As shown in 3, a "smooth" (rapidity) distribution, which does not show any fluctuations except for the statistical ones, has the property of $F_{q}(\delta y)$ 

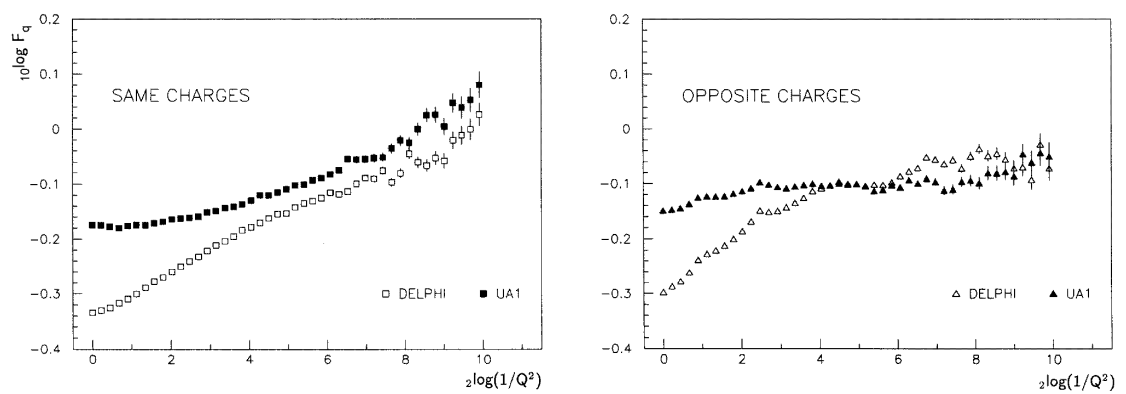

Figure 1. Comparison of density integrals for $q$ ॠ 2 as a function of $2 \log \left(1 / Q^{2}\right)$ for $\mathrm{e}^{+} \mathrm{e}^{-}$ (DELPHI) and hadron-hadron collisions (UA1).

being independent of the resolution $\delta y$ in the limit $\delta y \rightarrow 0$. On the other hand, if self-similar dynamical fluctuations exist, the $F_{q}$ obey the power law

$$
F_{q}(\delta y) \propto(\delta y)^{-\phi_{q}}, \quad(\delta y \rightarrow 0) .
$$

The powers $\phi_{q}$ (slopes in a double-log plot) are related 6 to the anomalous dimensions $d_{q}=\phi_{q} /(q-1)$, a measure for the deviation from an integer dimension. Equation $(8)$ is a scaling law since the ratio of the factorial moments at resolutions $L$ and $\ell, R=F_{q}(\ell) / F_{q}(L)=(L / \ell)^{\phi_{q}}$, only depends on the ratio $L / \ell$, but not on $L$ and $\ell$, themselves.

The scaling behavior is related to the physics of fractals. Fractal behavior is indeed expected for multiparticle production both from QCD branching and from a QCD phase transition.

\section{The State of the Art on Power-Law Scaling (Intermittency)}

2.1 Existence of scaling: The suggestion that the $F_{q}$ or $K_{q}$ might show powerlaw behavior has spurred a vigorous experimental search and (more or less) linear dependence of $\ln F_{q}$ on $-\ln \delta \Omega$ has been found in all types of collisions. 3 Recent $\mathrm{e}^{+} \mathrm{e}^{-}$results will be presented here by De Wolf.

Of particular interest is a comparison of hadron-hadron to $\mathrm{e}^{+} \mathrm{e}^{-}$results in terms of same and opposite charges of the particles involved. This comparison has been done for UA1 and DELPHI data in $\mathrm{U}$ and is shown in Fig. 1 for $q=2$. Important differences between UA1 and DELPHI can be observed:

For relatively large $Q^{2}\left(>0.03 \mathrm{GeV}^{2}\right)$, where Bose-Einstein effects do not play a major role, the $\mathrm{e}^{+} \mathrm{e}^{-}$data increase much faster with increasing ${ }_{2} \log \left(1 / Q^{2}\right)$ than the hadron-hadron data. The increase in this $Q^{2}$ region is very similar for same and for opposite charges. At small $Q^{2}$, however, the 
$\mathrm{e}^{+} \mathrm{e}^{-}$results approach the hadron-hadron results and the increase is faster for same than for opposite charges. So, at least two processes are responsible for the power-law behavior: Bose-Einstein correlation at small $Q^{2}$ following the evolution of jets at larger $Q^{2}$.

As such, Bose-Einstein correlations from a static source are not powerbehaved. A power law is obtained $\mathrm{l}$ if $\mathrm{i}$ ) the size of the interaction region is allowed to fluctuate, and/or ii) the interaction region itself is a self-similar object.

2.2 Multifractal or Monofractal: Fractals with all $d_{q}$ equal are called monofractals, those with $d_{q}$ depending on $q$ are called multifractals. A second-order phase transition (e.g. from a QGP) would give rize to a monofratal behavior of particle production, a cascade process (as e.g. QCD branching) leads to a multifractal behavior. Even though the $d_{q}$ and their $q$ dependence are found to be largest in $\mathrm{e}^{+} \mathrm{e}^{-}$and smallest in heavy-ion collisions, so far all types of collisions (including AA collisions) exhibit multifractal behavior. We shall hear more about monofractal behavior from N. Antoniou.

2.3 Self-similar or Self-affine Fractal: If the power law (8) holds when pspace is partitioned by the same factor in different directions, the fractal is called self-similar. If, on the other hand, (8) holds and only holds when space is partitioned by different factors in different directions, the corresponding fractal is called self-affine. 11

Self-affinity can be characterized by the Hurst exponents $11 H_{i j}=$ $\ln M_{i} / \ln M_{j} \quad\left(0 \leq H_{i j} \leq 1\right)$ with $M_{i}\left(i=1,2,3 ; \quad M_{1} \leq M_{2} \leq M_{3}\right)$ being the partition numbers in the phase-space variables $p_{i}$. The $H_{i j}$ can be obtained from the saturation of the one-dimensional $\ln F_{2}\left(\delta p_{i}\right)$ distributions.

For $h h$ collisions, the $H_{i j}$ for longitudinal-transverse combinations is indeed smaller than unity for NA22 22 and NA27 13 , while it is consistent with unity within the transverse plane (see Wang Shaoshun for hh and Chen Chang for first results on $\mathrm{e}^{+} \mathrm{e}^{-}$collisions)

2.4: QCD + LPHD have been quite successful 14 in the description of singleparticle $\xi$ distributions and the energy evolution of its maximum position $\xi^{*}$ and integral $n$. According to Eqs. (11) and (2), these correspond to $q=1$ only and, therefore, neglect any vital information on particle correlations.

a. High-Order Correlations: The ratio $H_{q}=K_{q} / F_{q}$ reflects the genuine $q$-particle correlation relative to the $q$-particle density. They have been calculated for gluons at different orders of QCD.15 For NNLLA, $H_{q}$ decreases to a negative first minimum for $q=5$ and shows quasi-oscillation around 0 for $q>5$. Assuming the validity of LPHD, such a behavior is also expected 
for the charged particles. For $\mathrm{e}^{+} \mathrm{e}^{-} \rightarrow$ hadrons at $\sqrt{s}=M_{\mathrm{Z}}$, SLD 10 indeed observed such a behavior. However the same behaviour is observed in hh collisions between 20 and $900 \mathrm{GeV} 17$ and exen in hA and AA collisions. 18

An L3 analysis of sub-jet multiplicities 19 reveals that this behavior appears only for energy scales $\lesssim 100 \mathrm{MeV}$, far away from the perturbative region. Furthermore, similar oscillatory behavior is observed for a large variety of MC models, including independent fragmentation. One is tempted to conclude, that at present energies, the oscillations of $H_{q}$ are unrelated to the behavior predicted by the NNLLA calculations. They may be related to the energy conservation first approximated in NNLLA, but what do we have to think of a theory approximating energy conservation only in such a high order of perturbative expansion?

The oscillations are reproduced by a superposition of 3 negative binomials 20 corresponding to two-jet, mercedes-like three-jet and intermediate events, respectively.19 So, the oscillations originate from an interplay between hard (jet topology) and soft (fragmentation) phenomena.

b) Local fluctuations: In perturbative QCD, the fractal strycture of jets follows from parton branching and the $\phi_{q}$ are directly related 9 to the anomalous dimension $\gamma_{0}=\left(6 \alpha_{s} / \pi\right)^{1 / 2}$, at lowest order via $d_{q}=D-\gamma_{0}(q+1) / q$, where $D$ is the topological dimension of the analysis and the second term corresponds to the Rényi dimensions $D_{q}$ of the fractal.

Analytical QCD predictions have indeed been given for fluctuations in angular intervals. 6 Assuming LPHD once again these predictions can be compared to experimental data. DELPHI 21, L3 22 and ZEUS 23 show that the predictions disagree with the data. Energy-conservation effects are indeed sizeable.24 Recent ZEUS results will be presented here by L. Zawiejski and the state of understanding will be summarized by W. Ochs.

2.5 Event-to-Event Fluctuations, Erraticity, Voids and Entropy: The $F_{q}$ as defined in (6) contain averages over all bins within one event and over the whole event sample. Event-to-event fluctuations lost in this procedure can be quantified 25 by defining the $F_{q}^{e}$ per event and determining moments of the distribution $P\left(F_{q}^{e}\right)$ as $C_{q, p}=\left\langle\left(F_{q}^{e}\right)^{p}\right\rangle /\left\langle F_{q}^{e}\right\rangle^{p}$. However, in an analysis of the influence of statistical fluctuations, 26 it turned out that these completely dominate, even in heavy-ion collision.

When the event multiplicity is low, the gaps between neighboring particles carry more information than multiplicity spikes. The method has therefore been extended to measure the rapidity gaps (raids).27 Experimentally the erraticity measures have been studied on NA27 28 (see Wang Shaoshun).

A method most sensitive to the maximum of the probability distribution 
is a measure of entropy suggested in 29.

Furthermore, results based on event-to-event $p_{\mathrm{t}}$ fluctuations will be presented here by Bai Yuting and T. Sugitate.

\section{Bose-Einstein Correlations}

3.1 Alternative Views: As can be seen on Fig. 1, BEC play a role at small $Q$, both in hh and $\mathrm{e}^{+} \mathrm{e}^{-}$collisions. Conventionally, they are studied within intensity interferometry 30 Basic ingredients are incoherent (chaotic) boson emission from a spherically symmetric static source and production amplitudes independent of momentum. Undere these conditions,

$$
\begin{aligned}
& R_{2} \equiv 1+K_{2}=1+|\tilde{\rho}(Q)|^{2} \\
& R_{3}=1+\left|\tilde{\rho}_{12}\right|^{2}+\left|\tilde{\rho}_{23}\right|^{2}+\left|\tilde{\rho}_{31}\right|^{2}+2 \operatorname{Re}\left(\tilde{\rho}_{12} \tilde{\rho}_{23} \tilde{\rho}_{31}\right),
\end{aligned}
$$

where $\tilde{\rho}(Q)$ is the Fourier transform of the space-time density of boson emitters. L. Gurvits will show us, however, how far astrophysics has developed away from intensity to amplitude interferometry, in the meantime.

A formalism particularly handy for the fully-dimensionaltreatment of a dynamical emitter is the so-called Wigner-function formalism 31 This is based on the emission function $S(x, p)$, a covariant Wigner-transform of the source density matrix. $S(x, p)$ can be interpreted as a quantum-mechanical analogue of the classical probability that a boson is produced at a given space-time point $x=(t, \mathbf{r})$ with a given momentum-energy $p=(E, \mathbf{p})$ (see T. Csörgö).

Alternatively, BEC are introduced into string models. 32 An ordering in space-time exists for the hadron momenta within a string. Bosons close in phase space are nearby in space-time and the length scale measured by BEC is not the length of the string, but the distance in boson-production points for which the momentum distributions still overlap (B. Andersson).

3.2 Recent experimental results: Bose-Einstein correlations are by now a well established effect in the hadronic final states of $\mathrm{Z}$ and $\mathrm{W}$ decay (intra-W BEC). 33 The important question is that of BEC between pions each originating from a different $\mathrm{W}$ in fully hadronic $\mathrm{W}^{+} \mathrm{W}^{-}$final states (inter-W BEC). If existent, such a correlation could, on the one hand, cause a bias in the mass determination of the $\mathrm{W}$. On the other, it could serve as a pion-interferometry laboratory for the measurement of the space-time development. The recent status of the search for inter-W BEC will be covered by J. van Dalen.

Detailed knowledge of intra-W BEC would be necessary to understand inter-W BEC. Limited statistics prevents that, but a large amount of high statistics information has recently been obtained for the $\mathrm{Z}$ and other reactions.

kittel: submitted to World Scientific on October 31, 2018 
For shortness, I am forced to refer to recent Zakopane lectures 34 on this topic and to limit myself to just listing the main results:

Bose-Einstein correlations exist also in $\mathrm{e}^{+} \mathrm{e}^{-}$collisions and DIS, with radius parameters independent of $\sqrt{s}$ and $Q^{2}$, respectively (E. De Wolf and L. Zawiejski).

The region of homogeneity is elongated along the evept axis.35 This is not reproduced by JETSET + LUBOEI, but expected from 32 . The short range of this region is evidence for a strong momentum-position correlation.

The correlator $K_{2}$ is steeper than Gaussian. 36.33 This is contrary to most of the assumptions and interpretations in the literature, but, again expected from 32 .

All radius parameters show a $1 / \sqrt{m}_{\mathrm{T}}$ dependence in all types of reactions. 37 This may be explainable already from the Heisenberg pripciple, 38 but is expected from an inside-outside cascade with transverse flow.39 More results from RHIC here from T. Sugitate.

In hh and $A A$ collisions, there is emidence for dilution of $K_{2}$ due to independence of overlapping mechanisms. 40 Search for a similar dilution due to overlap of string pieces in gluon jets will be presented by N. van Remortel.

The pion emission function in space-time has been extracted for hh and AA collisions 41 In the transverse plane, it is Gaussian for AA collisions but ring-shaped (!) for hh collisions. Study of the $\mathrm{Z}$ is underway (T. Csörgö).

For $\pi^{0} \pi^{0}$, according to the string model, the radius should be smaller than for charged pions. Wes Metzger will present the experimental evidence.

Genuine three-particle correlations contain additional information on a phase or, alternatively, on dilution of $K_{q}$ proportional to $N^{(1-q)}$ for $N$ independent sources. W. Metzger will give recent results on the Z.

Modelling these observations in a Monte Carlo code is a major challenge (see G. Wilk, K. Fiałkowski).

\section{Conclusion}

We need a model containing parton branching and energy conservation plus soft fragmentation to reproduce the fractal behavior of particle production. JETSET and ARIADNE are doing surprisingly well there, HERWIG a bit less. We further need the model to do away with old prejudice from conventional pion interferometry. A large amount of new information is (becoming) available on BEC at the Z. Models neglecting this information I would consider a loss of time. 


\section{References}

1. A.H. Mueller, Phys. Rev. D4, 150 (1971).

2. E.A. De Wolf et al, Phys. Rep. 270, 1 (1996).

3. A. Białas, R. Peschanski, Nucl. Phys. B273, 703 (1986); ibid. B308, 857 (1988).

4. P. Carruthers et al, Phys. Lett. B 54, 258 (1991).

5. P. Lipa et al, Phys. Lett. B285, 300 (1992).

6. P. Lipa, B. Buschbeck, Phys. Lett. B223, 465 (1989); R. Hwa, Phys. Rev. D41, 1456 (1990).

7. F. Mandl, B. Buschbeck in Proc. Cracow Workshop on Multiparticle Production, eds. A. Białas et al (World Scientific, Singapore, 1994) p.1.

8. A. Białas, Acta Phys. Pol. B23, 561 (1992).

9. G. Gustafson, A. Nilsson, Z. Phys. C52, 533 (1991); W. Ochs, J. Wosiek, Phys. Lett. B289, 159 (1992); ibid. 305, 144 (1993); Z. Phys. C68, 269 (1995); Y.L. Dokshitzer, I.M. Dremin, Nucl. Phys. B402, 139 (1993); Ph. Brax et al, Z. Phys. C62, 649 (1994).

10. W. Ochs, Phys. Lett. B247, 101 (1990); Z. Phys. C50, 339 (1991).

11. Y.F. Wu, L.S. Liu, Phys. Rev. Lett. 70, 3197 (1993); Y.F. Wu et al, Phys. Rev. D51, 6576(1995); Liu Feng et al, Phys. Rev. D59, 114020 (1999).

12. N.M. Agababyan et al (NA22), Phys. Lett. B382, 305 (1996); ibid. B431, 451 (1998).

13. Wang Shaoshun et al, Phys. Lett. B410, 323 (1987).

14. I.M. Dremin, J.W. Gary, Phys. Rep. 349, 301 (2001).

15. I.M. Dremin, Phys. Lett. B313, 209 (1993); I.M. Dremin, V.A. Nechitaľlo, JETP Lett. 58, 811 (1993); I.M. Dremin, Physics-Uspekhi 37, 715 (1994).

16. K. Abe et al (SLD Collab.), Phys. Lett. B371, 149 (1996).

17. I.M. Dremin et al, Phys. Lett. B336, 199 (1994); N. Nakajima et al, Phys. Rev. D54, 4333 (1996); Wang Shaoshun et al, Phys. Rev. D56, 1668 (1997).

18. A. Capella et al, Z. Phys. C75, 89 (1997); I.M. Dremin et al, Phys. Lett. B403, 149 (1997).

19. P. Achard et al (L3), subm. to Phys. Lett. B.

20. A. Giovannini et al, Phys. Lett. B374, 231 (1996); B388, 639 (1996).

21. P. Abreu et al (DELPHI Collab.), Phys. Lett. B457, 368 (1999).

22. M. Acciari et al (L3 Collab.), Phys. Lett. B428, 186 (1998).

23. S. Chekanov et al (ZEUS), Phys. Lett. B510, 36 (2001).

24. J.-L. Meunier, R. Peschanski, Z. Phys. C72, 647 (1996). 
25. Z. Cao, R.C. Hwa, Phys. Rev. Lett. 75, 1268 (1995); Phys. Rev. D53, 6608 (1996); ibid. D54, 6674 (1996); R.C. Hwa, Acta Phys. Pol. B27, 1789 (1996).

26. Liu Lianshou et al, Science in China A30, 432 (2000); Fu Jinghua et al, Phys. Lett. B472, 161 (2000); Liu Fuming et al, Phys. Lett. B516 (2001) 293.

27. R.C. Hwa, W. Zhang, Phys. Rev. D62, 0140003 (2000); R.C. Hwa, Y. Wu, Phys. Rev. D60 097501 (1999).

28. Wang Shaoshun, Wu Chong, Phys. Lett. B505, 43 (2001).

29. A. Białas et al, Acta Phys. Pol. B30, 107 (1999); A. Białas, W. Czyż, Phys. Rev. D61 074021 (2000); Acta Phys. Pol. B31, 687 (2000).

30. R. Hanbury Brown, R.Q. Twiss, Phil. Mag. 45, 663 (1954).

31. H. Gyulassy et al, Phys. Rev. C20, 2267 (1979); S. Pratt et al, Phys. Rev. C42, 2646 (1990); S. Chapman, U. Heinz, Phys. Lett. B340, 250 (1994).

32. B. Andersson, W. Hofmann, Phys. Lett. B169, 364 (1986); B. Andersson, M. Ringnér, Phys. Lett. B421, 283 (1998) and Nucl. Phys. B513, 627 (1998); Š. Todorova-Nová, J. Rameš, Strasbourg preprint IReS97-29.

33. P.D. Acton et al (OPAL), Phys. Lett. B267, 143 (1991); P. Abreu et al (DELPHI), Phys. Lett. B286, 201 (1992); Z. Phys. C63, 17 (1994); D. Decamp et al (ALEPH), Z. Phys. C54, 75 (1992); M. Acciari et al (L3), Phys. Lett. B493, 233 (2000).

34. W. Kittel, hep-ph/0110088, Acta Phys. Pol. (to be published).

35. M. Acciarri et al (L3), Phys. Lett. B458, 517 (1999); P. Abreu et al (DELPHI), Phys. Lett. B471, 460 (2000); G. Abbiendi et al (OPAL), Z. Phys. C16, 423 (2000).

36. N.M. Agababyan et al (NA22), Z. Phys. C59, 405 (1993); N. Neumeister et al (UA1), Z. Phys. C60, 633 (1993).

37. G. Bearden et al (NA44), Phys. Rev. C58, 1656 (1998); B. Lörstad, O.G. Smirnova, Proc. 7th Int. Workshop on Multiparticle Production, Nijmegen, eds. R.C. Hwa et al. (WSPC, Singapore, 1997) p.42.

38. G. Alexander et al, Phys. Lett. B452, 159 (1999); G. Alexander, Phys. Lett. B506, 45 (2001).

39. A. Białas, K. Zalewski, Acta Phys. Pol. B30, 359 (1999).

40. B. Buschbeck et al, Phys. Lett. B481, 187 (2000); Nucl. Phys. B (Proc. Suppl.) 92, 235 (2001).

41. N.M. Agababyan et al (NA22), Phys. Lett. B422, 359 (1998); A. Ster et al, Nucl. Phys. A661, 419 (1999); R. Hakobyan in Proc. XXX Int. Symp. on Multiparticle Dynamics, eds. R. Csörgő et al (WSPC, Singapore, 2001) p.331. 